\title{
Multimodal imaging of congenital hypertrophy of retinal pigment epithelium (chrpe) lesions at different presentations.
}

\author{
Vishal Raval*, Samrrudhi Dalal, Shreyansh Doshi, Taraprasad Das \\ Department of Ophthalmology, LV Prasad Eye Institute, Vijayawada, India
}

\begin{abstract}
Purpose: To describe the clinical presentation, fundus autofluorescence (FAF), optical coherence tomography (OCT) and OCT angiography (OCT-A) features of all three types of CHRPE: a solitary type, grouped configuration "bear tracks" and multifocal type with the systemic association. Methods: A comprehensive eye examination included measurement of presenting and best-corrected vision, applanation intraocular pressure (IOP), slit-lamp biomicroscopy and dilated fundus examination. The retinal imaging included fundus photography, FAF, OCT, and OCT-A. Results: The study included retinal imaging of 3 varieties of CHRPE seen in 3 patients on routine eye examination. In all patients vision was 20/20, N6 in both eyes, and IOP was 14-18 mmHg. Fundus showed bilateral, multifocal, flat, pigmented retinal lesions (Case 1), solitary, the well-circumscribed lesion (Case 2) and multiple small, flat, pigmented lesions (Case 3). FAF in all 3 variants showed hypoautofluorescence corresponding to the area of the lesion with isolated areas of is fluorescence in areas of lacunae. OCT in CFase 1 and 2 showed increased reflectance of RPE layer with outer retinal layer abnormalities whereas in Case 3 showed normal RPE layer. OCT (Case 1) also revealed thickening of RPE associated with the intraretinal extension. OCTA of outer retinal layers in Case 1 and 2 showed vascular attenuation over the CHRPE lesion with hyporeflective areas whereas in Case 3 it was normal. Conclusion: A careful clinical examination with multimodal imaging helps to differentiate a benign variant of CHRPE from its counterpart associated with systemic features.
\end{abstract}

Keywords: Congenital hypertrophy of retinal pigment epith elium, optical coherence tomography, autofluorescence, optical coherence tomography angiography.

Accepted on April 10, 2019

\section{Introduction}

Congenital Hypertrophy of The Retinal Pigment Epithelium (CHRPE) is congenital hamartomas of Retinal Pigment Epithelium (RPE) which can present as flat, well-demarcated hyperpigmented lesion located at the level of the RPE [1-3]. They are seen in the normal population with a prevalence of $1.2 \%$ and are detected on routine retinal examination [4]. It can present either as a solitary lesion or as multifocal lesions known as congenital grouped pigmentation or "bear tracks" [3]. While there are reports of association of familial adenomatous polyposis and Gardner syndrome with similar bilateral, multiple, irregular fundus lesions, the typical solitary and multifocal forms of CHRPE are not associated with these diseases [5]. We describe the details of retinal imaging of all 3 varieties of CHRPE seen in 3 different patients on routine eye examination. The retinal imaging included fundus photography, Fundus Autofluorescence (FAF), Optical Coherence Tomography (OCT) and OCT angiography (OCT-A) in addition to clinical examination including indirect ophthalmoscopy and slit-lamp biomicroscopy. In all patients the best-corrected vision was 20/20, N6 in both the eyes and the applanation intraocular pressure was within normal limits (14 to $18 \mathrm{~mm} \mathrm{Hg}$ ).

\section{Case Reports}

\section{Case 1}

Bilateral, multifocal, flat, pigmented retinal lesions ( $>4$ in each eye) were seen in pseudophakic eyes of a 64-year old man. These lesions were located in mid-periphery; they were dark pigmented, oval to circular in shape with slightly irregular margins, surrounded by a hypopigmented halo and central lacunae (Figure 1 and 1b). FAF showed hypoautofluorescence corresponding to the pigmented areas of the lesion and few isolated areas of isofluorescence corresponding to the areas of lacunae (Figure 1c and 1d). OCT through the pigmented lesion showed increased reflectance of RPE with intraretinal extension, loss of photoreceptor layer and disruption of outer retinal layers; the OCT through the lacunae area showed the absence of RPE layer (Figure 1e and 1f). The choroid and choriocapillaris layer underneath the lesion appeared normal. OCT-A in the outer retinal slab showed vascular attenuation over the CHRPE lesion with hyporeflective areas outlining the area of the lesion with few hyper-reflective areas within it corresponding to the lacunae (Figure $1 \mathrm{~g}$ and $1 \mathrm{~h}$ ). The choriocapillaris slab in the area of lesion was blocked due to the overlying hyporeflective area.

The systemic workup with a gastroenterologist confirmed the presence of multiple polyps in the large intestine on colonoscopy. 

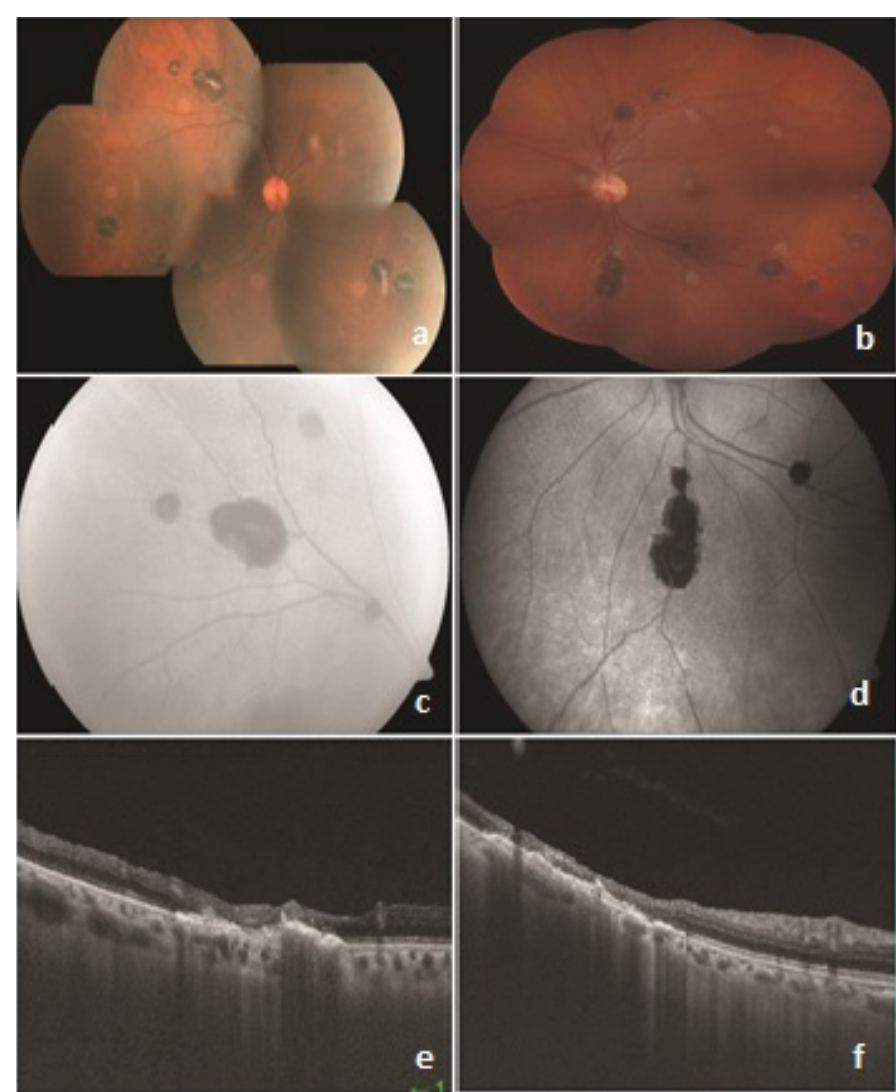

b
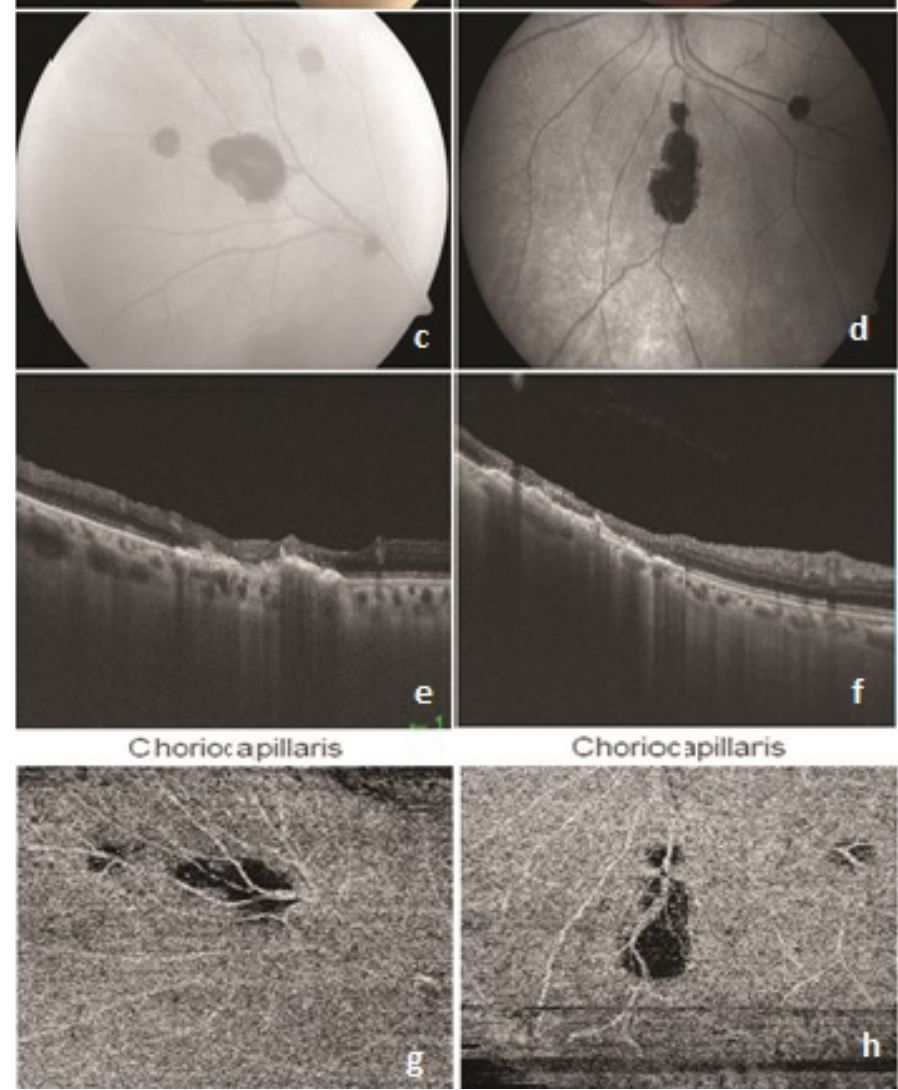

$\mathbf{f}$

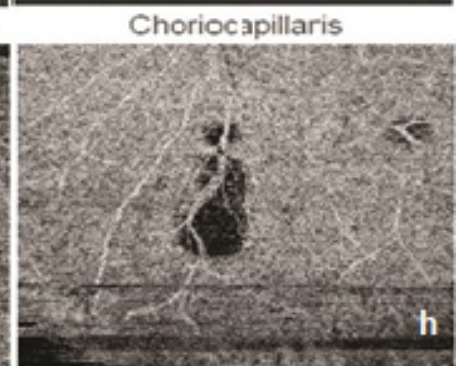

Figure 1. Colour fundus photography showed multiple, bilateral, flat, pigmented retinal lesions in mid-periphery with slightly irregular margins and, surrounded by hypopigmented halo and central lacunae (Figure 1a and 1b). Fundus Autofluorescence (FAF) showed hypoautofluorescence corresponding to the pigmented areas of the lesion and few isolated areas of isofluorescence corresponding to the areas of lacunae (Figure $1 c$ and 1d). Optical Coherence Tomography (OCT) through the pigmented lesion showed high reflective RPE layer with intraretinal extension and disruption of outer retinal layers; OCT through the lacunae area showed absence of RPE layer (Figure le and 1f). OCT-Angiography in the outer retinal slab showed vascular attenuation over the lesion with signal void areas outlining the area of lesion with few hyper-reflective areas within it corresponding to the lacunae (Figure $1 \mathrm{~g}$ and $1 \mathrm{~h}$ ).

This conformed to the diagnosis of multifocal CHRPE with familial adenomatous polyposis. While we advised the family members for a check-up of the gastrointestinal system, we requested our asymptomatic patient regular annual follow up.

\section{Case 2}

A solitary, well circumscribed, approximately 8-10 DD size, flat hyperpigmented lesion located in the superotemporal quadrant of the retina was detected in a 58-year old woman (Figure 2a). There was surrounding depigmented halo corresponding to RPE atrophy. The retinal vessels were crossing over the lesion with few areas of lacunae in the lesion. The FAF showed hypoautofluorescence with few areas of isofluorescence in the center (Figure 2b). The OCT through the pigmented lesion showed thinning of inner as well as outer retinal layers with increased reflectance of RPE (Figure 2c). Atrophied retinal layers were visible through the depigmented halo. The choroid and choriocapillaris could not be commented because of increased shadowing from thickened RPE, though the choroidal layer appeared normal at the margins of the lesion. OCT-A in the outer retinal slab showed vascular attenuation over the CHRPE lesion with hyporeflective areas outlining the area of the lesion (Figure 2d). The choriocapillaris slab in the area of lesion was blocked due to an overlying hyporeflective area with few areas of hyper-reflective lesion corresponding to areas of lacunae (Figure 2e). A diagnosis of solitary CHRPE was made and we requested him annual review to document future morphological changes in the lesion.

\section{Case 3}

Multiple small (2 $\mathrm{mm})$, flat, pigmented lesions located in the inferior quadrant were seen in a 14-year-old girl. These lesions were arranged in a pair with the lesion size gradually increasing radially from the optic disc resembling footprints of a bear track (Figure 3a). There was the absence of surrounding depigmented halo or lacunae within these lesions. FAF showed marked hypoautofluoresence which outnumbered clinically identifiable lesions (Figure 3b). The OCT scan through these lesions showed a near normal RPE layer without any hypertrophy or hyperplastic changes (Figure 3c). The overlying outer retinal layer and photoreceptor layer too were normal (Figure 3d). OCT-A through the lesions in both outer retina and choriocapillaris was normal without any microvasculature or structural alterations. In view of this benign condition, she was requested regular annual review (Table 1).

\section{Discussion}

Congenital hypertrophy of the retinal pigment epithelium is a benign lesion of the fundus. This term was first used by Buettner in 1975 [6]. Typical CHRPE consists of a single flat, round lesion, with sharply demarcated smooth or scalloped margins, which may vary in color from light brown to grey to black, and is nearly always unilateral [7]. There are 3 types of presentations: (1) solitary, the flat, well-demarcated, hyperpigmented lesion (most common), (2) a grouped configuration 'bear tracks' and (3) associated with a systemic association like Gardner's syndrome or familial adenomatous polyposis (most rare). Atrophied window-like defects called lacunae are often present. These lacunae are variable in size, shape and the percentage of the lesion which they cover. A ring of hypopigmentation is often found just inside the margin of the lesion producing a characteristic halo or double outline [7]. Typically, CHRPE has no clinical significance; however, the occurrence of multiple CHRPE lesions, which increases over time in number or size is often associated with the cancer syndrome such as the Familial Adenomatous Polyposis (FAP). It is an autosomal dominant disease caused by mutations in the Adenomatous Polyposis Coli (APC) gene [7]. 

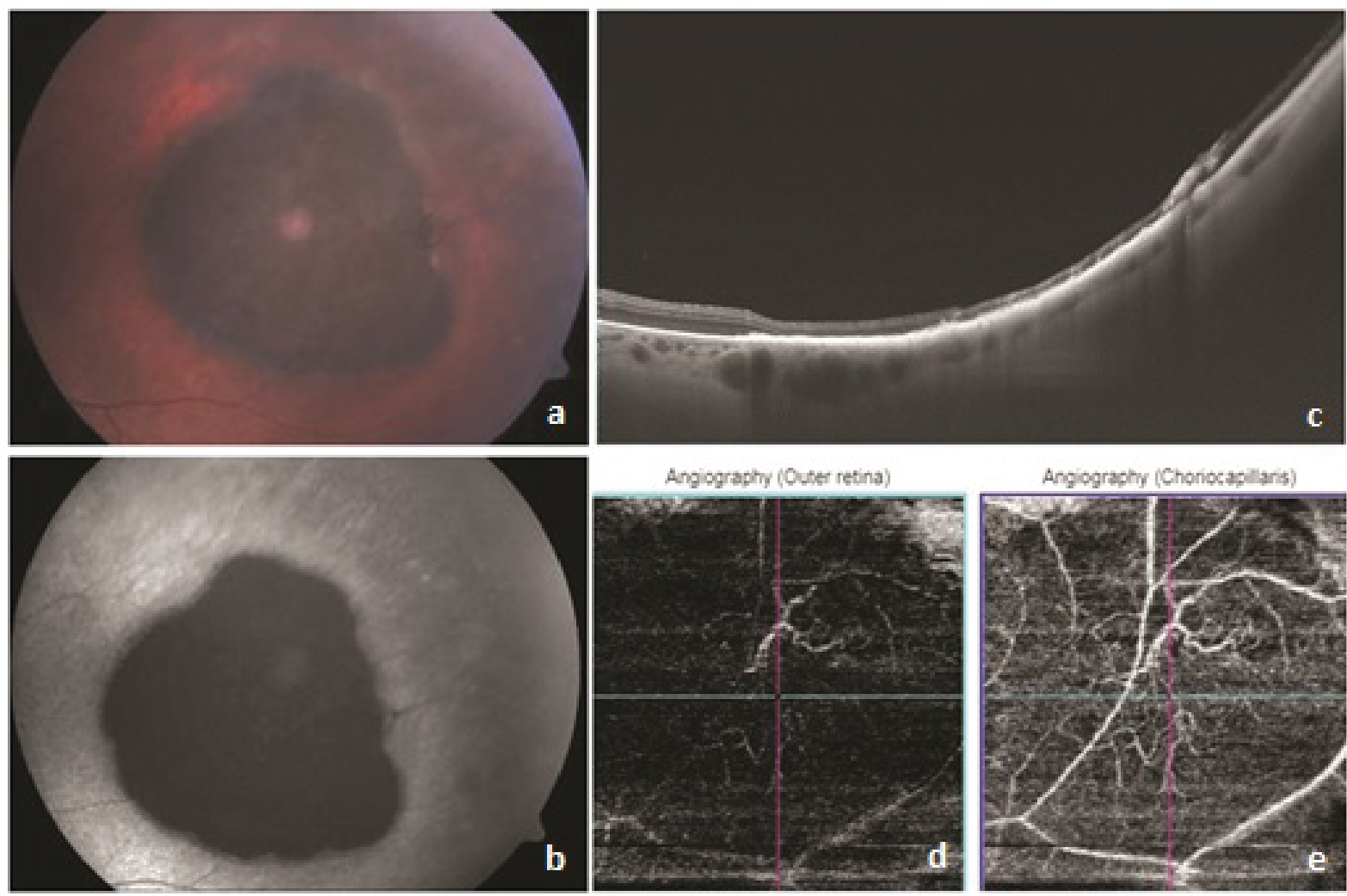

Figure 2. Colour fundus photography showed a focal, well circumscribed, flat hyperpigmented lesion in the superotemporal quadrant with surrounding depigmented halo. The retinal vessels were crossing over the lesion with few areas of lacunae in the lesion (Figure 2a). The FAF showed hypoautofluorescence with few areas of isofluorescence in the centre (Figure 2b). The OCT through the pigmented lesion showed increased reflectance of RPE with disruption of inner as well as outer retinal layers (Figure 2c). The choroidal layer appeared normal at the margins of the lesion. OCT-A in the outer retinal slab showed vascular attenuation over the CHRPE lesion with hyporeflective areas corresponding to lesion and few hyper-reflective areas corresponding to lacunae (Figure $2 d$ and $2 e$ ).
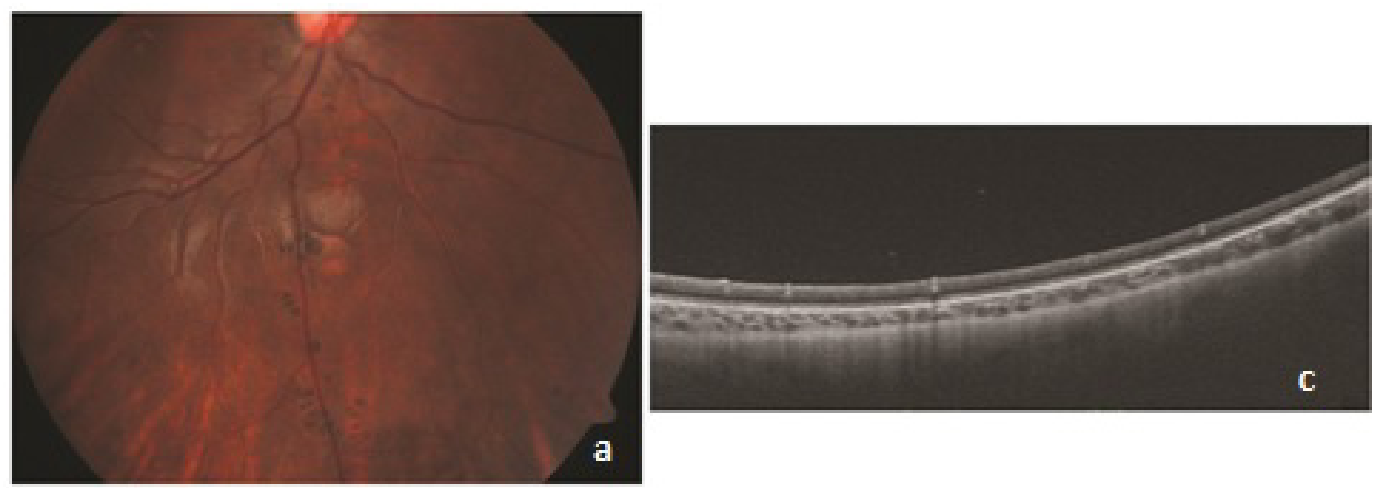

a
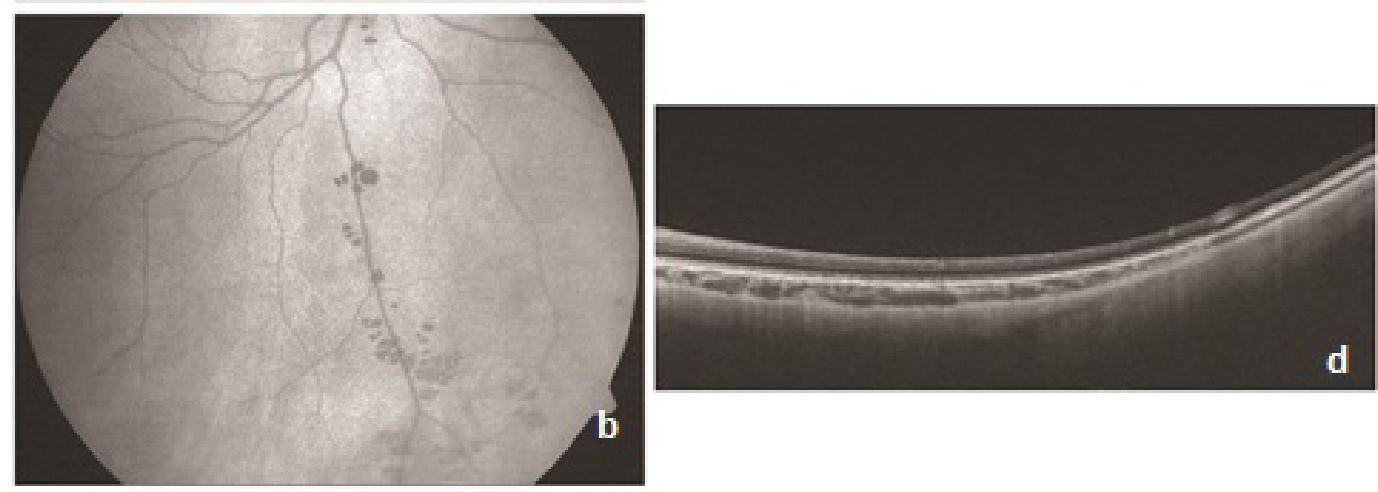

Figure 3. Colour fundus photography showed multiple, flat, pigmented lesions located in the inferior quadrant arranged in a pair resembling footprints of a bear track (Figure 3a). FAF showed marked hypoautofluoresence corresponding to areas of lesions (Figure 3b). The OCT scan showed a near normal RPE layer without any hypertrophy or hyperplastic changes (Figure 3c). The overlying outer retinal layer and photoreceptor layer too were normal (Figure 3d). 
Citation: Raval V, Dalal S, Doshi S, Das T. Multimodal imaging of congenital hypertrophy of retinal pigment epithelium (chrpe) lesions at different presentations. Ophthalmol Case Rep. 2019;3(1):1-4.

Table 1. The clinical features and various imaging modalities to differentiate various types of CHRPE lesions.

\begin{tabular}{|c|c|c|c|}
\hline CHRPE lesion & Solitary type & Multifocal grouped lesions (bear track) & Grouped lesions with systemic associations \\
\hline Fundus examination & $\begin{array}{l}\text { Flat, hyperpigmented lesion, mostly } \\
\text { located at the equatorial region. Areas } \\
\text { of lacunae are seen with sdepigmented } \\
\text { halo surrounding it. }\end{array}$ & $\begin{array}{l}\text { Multiple, small flat pigmented lesion located } \\
\text { at the posterior pole and gradually increases } \\
\text { towards the periphery. Resembles bear track } \\
\text { lesions. }\end{array}$ & $\begin{array}{l}\text { Bilateral, flat hyperpigmented lesions with irregular } \\
\text { margins. Areas of lacunae seen with overlying retinal } \\
\text { vasculature abnormality }\end{array}$ \\
\hline Fundus autofluorescence & $\begin{array}{l}\text { Hypoautofluorescence with few areas of } \\
\text { iso or hyper autofluorescence. }\end{array}$ & Hypoautofluorescent areas & $\begin{array}{l}\text { Hypoautofluorescence with few areas of iso or hyper } \\
\text { autofluorescence }\end{array}$ \\
\hline OCT & $\begin{array}{l}\text { Thickening of RPE layer with outer } \\
\text { retinal layers atrophy. }\end{array}$ & Normal RPE layer and outer retinal layers. & $\begin{array}{l}\text { Thickening and intraretinal extension of RPE layer } \\
\text { with outer retinal layers atrophy }\end{array}$ \\
\hline $\begin{array}{l}\text { OCT angiography (outer } \\
\text { retinal slab) }\end{array}$ & $\begin{array}{l}\text { Hyporeflective areas with areas of iso } \\
\text { reflective surrounding the lesion. }\end{array}$ & Normal vasculature & $\begin{array}{l}\text { Hyporeflective areas wth overlying retinal capillary } \\
\text { attenuation or dilatation. The surrounding choroidal } \\
\text { layers are normal. }\end{array}$ \\
\hline Systemic associations & None & None & $\begin{array}{l}\text { May be associated with familial adenomatous } \\
\text { polyposis or Gardner's syndrome }\end{array}$ \\
\hline Treatment & $\begin{array}{l}\text { Observation; rarely increase in size and } \\
\text { height may be noticed }\end{array}$ & Observation & $\begin{array}{l}\text { Familial screening and gastroenterologist review to } \\
\text { rule out table polypoidal lesions in the colon. }\end{array}$ \\
\hline
\end{tabular}

The typical fluorescein angiography changes in CHRPE are vascular non-perfusion areas with obliteration of the retinal capillary bed [8]. These morphological changes in retinal vasculature are most likely due to atrophy of outer retinal layers. Leakage at the margins of CHRPE lesion is seen occasionally [8]. In our series, we didn't perform FFA for lack of consent. However, OCT-A in the deep capillary slab showed vascular attenuation overlying the lesion in a solitary and multifocal group with hyporeflective areas; this represents the FFA appearance of capillary non perfusion areas. In the choriocapillary slab the surrounding choroidal vasculature was normal; we observed hyporeflective areas obscuring the choroidal layers and these were due to image artifact.

Shields et al. have described FAF in CHRPE; this consists of hypo-autofluorescence in the areas of CHRPE and hyperautofluorescence in the area of lacunae. The hypo-autofluorescee of CHRPE has been explained by the histopathologic absence of lipofuscin within the enlarged heavily and uniformly pigmented RPE cells [9], whereas hyper-autofluorescence in the areas of lacunae is probably due to the absence of RPE causing unmasking of background sclera autofluorescence.

Fung et al. have reported EDI-OCT features of CHRPE [10]. In their series, 16 of 18 patients showed hypertrophied RPE layer; the lacunae lesions were seen in 9 patients. The overlying retina above the lesion showed atrophy of the outer retinal layer, generally involving the outer nuclear layer to photoreceptors with the occasional presence of characteristic subretinal cleft at the area of absent photoreceptors. The underneath Bruch membrane and choriocapillary layers were unaffected. In our series, the OCT in the multifocal lesion had thickened RPE with intraretinal extension. We noted hypertrophied RPE layer with disruption of outer retinal layers and thinning of the photoreceptor layer in solitary form; there were no defect in outer retinal layers or photoreceptors layer in the 'bear track' lesions. Histopathology of the 'bear track' lesions has shown that the RPE cells retain their normal ellipsoid configuration without hypertrophy or hyperplasia [7].

\section{Conclusion}

The CHRPE are generally benign, but one must watch for occasional occurrence of bilateral multifocal lesions that could be associated with Gardner's syndrome or familial adenomatous polyposis. A careful clinical examination coupled with multimodal imaging described here help differentiate the benign from the lesions pointing to colorectal cancer.

\section{Funding}

Hyderabad Eye Research Foundation.

\section{References}

1. Shields CL, Mashayekhi A, Ho T, et al. Solitary congenital hypertrophy of the retinal pigment epithelium: Clinical features and frequency of enlargement in 330 patients. Ophthalmology. 2003;110:1968-76.

2. Shields JA, Shields CL. Intraocular tumors: An Atlas and Textbook. 3rd ed. Philadelphia: Wolters Kluwer. 2015:453-502.

3. Shields CL, Materin MA, Walker C, et al. Photoreceptor loss overlying congenital hypertrophy of the retinal pigmentepithelium by optical coherence tomography. Ophthalmology. 2006;113:661-65.

4. Coleman P, Barnard NA. Congenital hypertrophy of the retinal pigment epithelium: Prevalence and ocular features in the optometric population. Ophthalmic Physiol Opt. 2007;27:547-55.

5. Shields JA, Shields CL, Shah PG, et al. Lack of association between typical congenital hypertrophy of the retinal pigment epithelium and Gardner's syndrome. Ophthalmology. 1992;99:1709-13.

6. Buettner H. Congenital hypertrophy of the retinal pigment epithelium. Am J Ophthalmol. 1975;79:177-89.

7. Shields JA, Shields CL. Tumors and related lesions of the pigmented epithelium. Asia Pac J Ophthalmol. 2017;6:215-23.

8. Cohen SY, Quentel G, Guiberteau B, et al. Retinal vascular changes in congenital hypertrophy of the retinal pigment epithelium. Ophthalmology. 1993;100:471-74.

9. Shields CL, Pirondini C, Bianciotto C, et al. Autofluorescence of congenital hypertrophy of the retinal pigment epithelium. Retina. 2007;27:1097-1100.

10. Fung AT, Pellegrini M, Shields CL. Congenital hypertrophy of the retinal pigment epithelium: Enhanced depth imaging optical coherence tomography in 18 cases. Ophthalmology. 2014;121:251-56.

\section{*Correspondence to:}

Vishal Raval

Department of Ophthalmology

LV Prasad Eye Institute

Vijayawada, India

Tel: 08374588011

E-mail: drvishalraval@gmail.com 